\title{
12
}

\section{ONTOLOGICAL STRATIFICATION IN AN ECOLOGY OF INFOHABITANTS}

\author{
V.A. Abramov ${ }^{1}$, J.B.M. Goossenaerts ${ }^{1}$, P. De Wilde ${ }^{2}$, and L. Correia ${ }^{3}$ \\ ${ }^{1}$ Eindhoven University of Technology, the Netherlands \\ ${ }^{2}$ Imperial College of Science, Technology and Medicine, London, UK \\ ${ }^{3} \mathrm{New}$ University of Lisbon, Portugal \\ e-mail: J.B.M.Goossenaerts@tm.tue.nl
}

Abstract: This paper reports progress from the EEII research project where ontological stratification is applied in the study of openness. We explain a stratification approach to reduce the overall complexity of conceptual models, and to enhance their modularity. A distinction is made between ontological and epistemological stratification. The application of the stratification approach to agent system design is explained and illustrated. A preliminary characterization of the relevant strata is given. The wider relevance of this result for information infrastructure design is addressed: ontological stratification will be key to the model management and semantic interoperability in a ubiquitous and model driven information infrastructure.

Keywords: Ontological stratification, agent systems, semantic interoperability

\section{INTRODUCTION}

Agent system research and development has become an active field. There are many examples of using agent systems to solve tasks in so various areas as electronic commerce [1,2], control [3], communication and networks [4], resource management [5], search and information retrieval [6], etc. On the other hand, the novelty and at the same time immaturity of the existing results hinders introduction of agent systems into real life applications. One of the major reasons for that is the fact that most of the research efforts are concentrated on designing agents, components of agents, 
or agent systems. There is little time left for analysis of the properties of the designed systems.

Questions about such system properties as openness, robustness, stability, adaptability, scalability, should be answered before an agent system is deployed in industrial applications and infrastructures. However, only few research results exist that help to answer these questions qualitatively [7] and even less - quantitatively [3]. There is still a large gap between the number of developed agent systems and number of analysis methods and tools for their evaluation.

In order to address this gap the EEII project (Evolution and Ecology of Interacting Infohabitants, EU IST-1999-10304) is developing an ecological approach to modeling and analysis of such agent system properties as scalability, openness, stability, and adaptability. The methods applied within this approach include analytical estimations, discrete simulations of agent communities [8], and theoretical considerations regarding system design methods [9]. One of the methods developed is a macro-level ecological analysis method oriented on investigation of agent system openness. Essential part of this method is the practical application of stratification approach influenced by other work on piecemeal ontological commitment in a participative simulation environment [10] and by other multi-strata models [11]. A two-sided stratification approach, with ontological and epistemic stratification, is described in the next section. Then we describe the application of the approach to the agent system design. Finally we draw conclusions about the stratification approach and conjecture about its possible role within a ubiquitous and model driven information infrastructure [12].

\section{APPLYING STRATIFICATION}

\subsection{Ontological Stratification}

The concept of ontological stratification has been initially developed in the field of Formal Ontology [13]. In essence, ontological stratification is the partitioning of (conceptualization of) an information system's world into several disjoint spaces (or strata) according to the identity criteria used to conceptualize the entities in these spaces. The following set of spaces is proposed by Guarino (with examples of corresponding entities): static (a situation, a configuration), mereological (an amount of matter), physical: (a) topological (a piece of matter) and (b) morphological (a cubic block), functional (an artifact), biological (a human body), intentional (a person or a robot), social (a company). 
It is easy to see that the same object may belong to one or more spaces, e.g., an apple may be considered as (i) an amount of matter when we are concerned with transportation of apples, (ii) a food when we want to satisfy our hunger, (iii) a seed when we think about planting an apple tree, or (iv) a product when an apple goes to a market. In all of these cases, different aspects of the same apple are considered, and different laws and rules are applied: transportation of an apple is mostly concerned with the laws of physics, using it as a food - with the functional considerations, using it as a seed - with the biological laws, selling it on market - with the laws of economics in modern society.

\subsection{Epistemic Stratification}

In modern society, when acquiring disciplinary knowledge, abstraction is used to separate all the abovementioned domains. The partitioning and single-stratum in-depth study of topics accelerates knowledge creation within a particular domain (linked to a stratum) without necessary involvement of knowledge from all the other domains.

Investigation of ontological commitment for participative simulation has revealed a distinction between the being and becoming of entities as studied in ontology proper, and the concept-enabled analyses in various knowledge domains $[14,15]$. The conceptual models that underlie these analyses are usually also referred to as part of ontology, but conformance with the use of terms in philosophy would position the study of these models in epistemology, the theory of knowledge [16]. Hence the use of the term epistemic stratification to denote the diversity of specialized knowledge models that are used by specialists of several domains of expertise, e.g. ergonomy, logistics, physics, sociology.

\subsection{Stratification and Information Systems}

Complex information systems are often based on models that describe the application domain, its objects, processes, tasks as well as available knowledge related to the task solving. Ontological and knowledge models used in such systems are often related to several spaces or strata in the Formal Ontology sense. Therefore it is reasonable to apply the above stratification approach to these models in order to reduce their overall complexity and increase modularity. This would facilitate modification, extension and re-use of information system models.

In our work, we deal with ontology and knowledge models related to several domains of expertise, and apply the stratification approach to both of these models. 


\section{STRATIFICATION IN AGENT SYSTEM DESIGN}

In our current research work, we are developing an agent system that is to be used within a complex domain. The system has to deal with different objects such as a physical object, a network node, a software agent, a person, etc. The system is distributed and networked. It should be used by many users to support their individual task-solving activities. Typical tasks are participation in distributed trade, in supply networks, etc.

The main components of the system are user (someone, typically a human, who uses other components of the system to solve its tasks), agent (someone, typically a software agent, who assists its user in solving tasks), and object (object of task).

These system components naturally belong to several different spaces. By distinguishing and specifying their involvement in specific spaces we can get a more clear description of the overall system. Within each space, more clear and compact specifications are possible of the tasks involving entities present in the space. For example, a (sub)-task of physical transportation of goods is mostly described in terms of entities of physical space. In contrast, a (sub)-task of demand-request matching involves only entities of conceptual space.

Finally and most important for our current research, the ontological stratification allows us to clearly distinguish, model and evaluate different types of agent system openness. For example, we can separately model and study information openness, conceptual openness, intentional openness, etc.

\subsection{The stratified agent system model}

We consider the agent system consisting of the following high-level types of components: user, agent and object. Components are complex and composite; we consider their sub-components or aspects to be part of the several distinct spaces. Each sub-component is located in one of the spaces (e.g., think of location in the physical space, network space, etc.) and is related to other entities of the same space in different ways.

Clearly, a product and a client are entities that should belong to the social space, because these entities only emerge in the context of social interactions. On the other hand, an apple is just a physical entity. An apple can as well be a food, or a product, but these entities belong to other spaces - functional and social correspondingly. There is a choice: either to consider such entities as an apple, a food, and a product as a single object or to admit xistence of three different (though dependent) entities. Together with Guarino, we accept the latter choice. As a result, the components of our agent system consist of interdependent sub-components in several spaces. In 
Figure 1, this way of ontological stratification is illustrated with concepts of the generic agent system model.

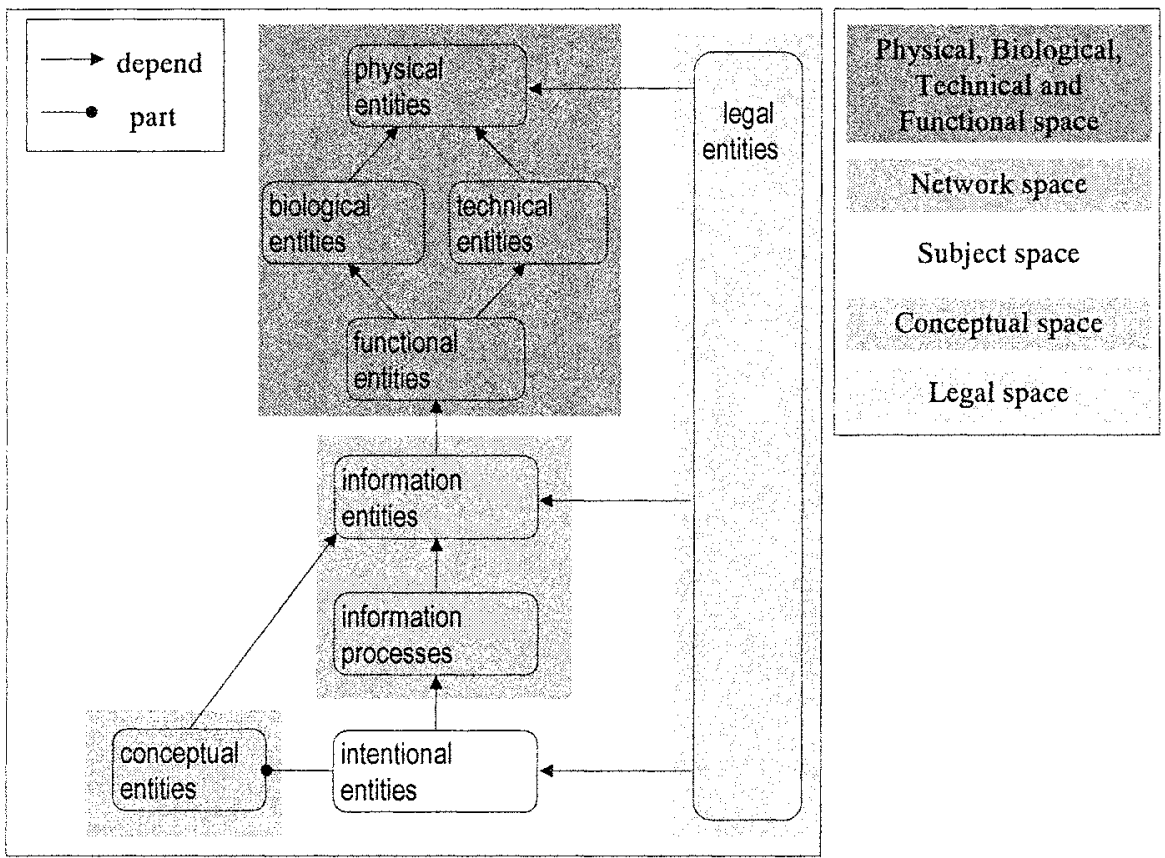

Figure 1. Stratification of generic agent system ontology

In the design of our test agent system, only part of the depicted spaces is used as described here. The subject space concerns the component's aspect of having certain goals, and being able to act intentionally. Clearly, users and agents have this aspect and therefore have presence in this space. The legal space contains legal aspects of the system components and legal relations among them. For example, user can own some property. Users and objects have presence in this space. The conceptual space contains all the concepts of an applied domain that are used by all the active components of the system. The network space consists of a number of interconnected nodes of an information network. Everything in this space has a network address, i.e., every sub-component of the agent system's components resides at some node. Users, agents and (information) objects have presence in this space. The physical space is organized by a number of geographically distributed sites that are interconnected by transportation links. Each site is associated with a unique geographical address. Every component that has presence in this space has an address, i.e., at any point of time it must be at a unique site. Users, agents and objects will have their presence in PS. Table 1 summarizes the presence of system components in described spaces: 
Table 1. Presence of components in different spaces

\begin{tabular}{|c|c|c|c|}
\hline Space & User & Agent & Object \\
\hline Subject space & subject & subject & \\
\hline Legal space & legal body & & legal body \\
\hline Conceptual space & concept(s) & concept(s) & \\
\hline Network space & $\begin{array}{l}\text { information } \\
\text { body }\end{array}$ & information body & $\begin{array}{l}\text { information body } \\
\text { (inf. objects only) }\end{array}$ \\
\hline Physical space & $\begin{array}{l}\text { physical } \\
\text { body }\end{array}$ & $\begin{array}{l}\text { physical body (variable for mobile } \\
\text { agents, invariable otherwise) }\end{array}$ & physical body \\
\hline
\end{tabular}

\subsection{An application scenario: the trading task}

An agent system simulating a distributed market place has been developed using the stratified ontology. In the system, there are a number of users who produce and consume some products. The users are distributed in the physical space. Users are interested in buying some products that other users have. Also, they want to sell their own products to earn money. A typical user task is to buy or sell specific amount of certain product. There are many types of products being traded by the users. These types are organized by individual users into partial classifications. All these classifications are individual, not precise and may partially overlap each other. Under these circumstances, the task of the agent system and each of the agents is to satisfy interests of the users.

In order to solve this task, one or more static and mobile agents may be employed by users. Interacting in the network space, the agents find trade partners, products, negotiate deals, sign and implement contracts. The trading process involves making agreement on the object of trade (ontology negotiation - each agent has its own applied ontology describing the types of products it can deal with) and price agreement (price negotiation). The ontology negotiation is an inherently error-prone process since agents do not share the standard ontology.

The crucial part of the experiment with the system is the study of the agent openness. The agents are provided with certain capabilities that can make them open when these capabilities are utilized. In particular, an agent can share parts of its experience with other agents (information openness). Also, an agent can receive from other agents and adopt concepts about new objects in the environment (conceptual openness). It can recognise those objects when familiar with the concepts. Finally, an agent can choose to share its task-solving intentions with (partner) agents (intentional openness). There are several more types of openness that have been identified in our research. At the moment, the agent system simulations provide a way to study the three types of openness mentioned above. When doing the simulations, we measure individual and population-level performance of 
agents and relate it to the level and type of agent openness. In Figure 2 the screenshots of the running simulation are presented.

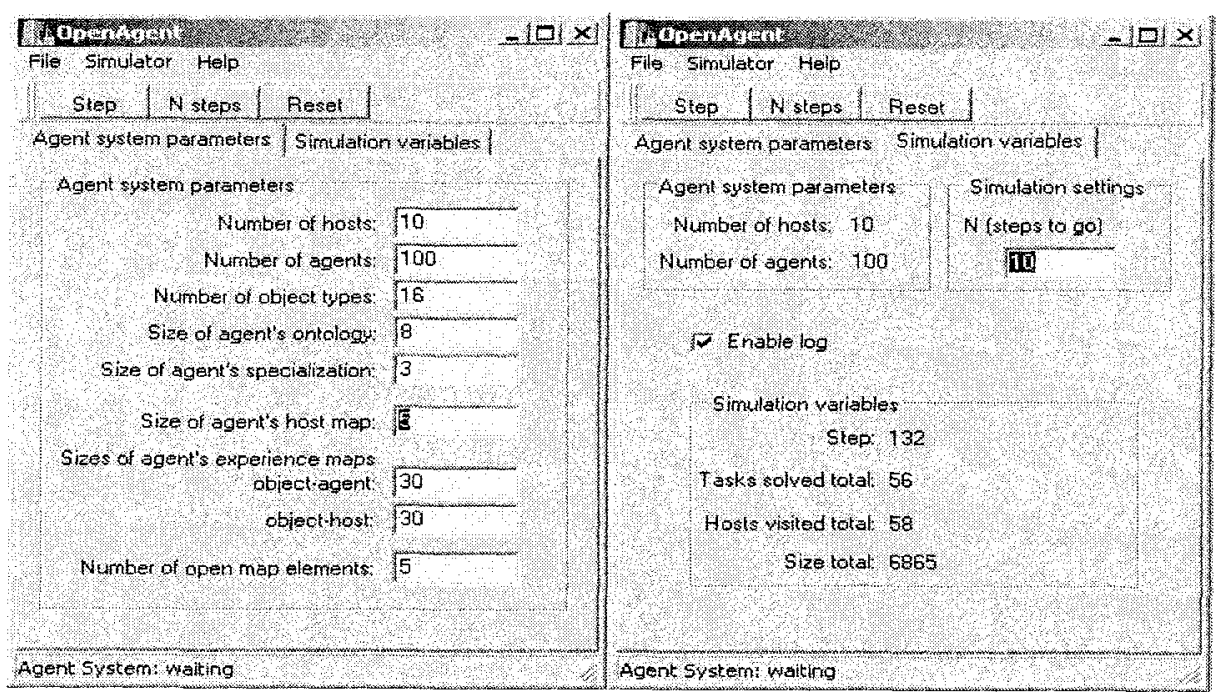

Figure 2. Agent system running an openness-related experiment

During the development of the agent system, a clear advantage has been identified of using a stratified conceptual model of the system. The fact that the key components of the system have been described as sets of subcomponents and that all spaces have been separately specified, has increased the system modularity. It has become easily possible to modify the structure of each of the spaces (for example, connectivity model of the network space, or the structure of the conceptual space) without affecting the other spaces. It has been also possible to change the laws governing the entities behaviour in each of the spaces independently.

Separation of conceptual, information and intentional entities into individual spaces has made clear the distinction between the respective types of agent openness. It also made possible independent experiments with each of these openness types. At the current stage of our work, we conduct these experiments to measure dependence of agents' performance on their openness.

\section{CONCLUSIONS AND FUTURE WORK}

Ontological stratification of the agent system conceptual model has provided us with a better ground for experiments with the system for two main reasons: it provided more structured and modular description of the system thus enabling easier design modifications, and it gave us a way to 
clearly distinguish types of entities relevant to the openness-related experiments that we conduct with the system.

Ontological stratification in general promises to enable constructing better ontologies for the information systems and their infrastructure. Epistemic stratification recognizes that specialized knowledge models are required for different domains of expertise and allows for easier joint work of specialists from these domains on a single project.

Research continues on the application of the stratification approach in the architecting of a model driven information infrastructure, aiming for results on semantic interoperability and efficient model management.

\section{REFERENCES}

1. Chavez, A., and P. Maes (1996) Kasbah: An agent marketplace for buying and selling goods. Proc. $1^{\text {st }}$ Int. Conf. on the Practical Application of Intelligent Agents and MultiAgent Technology, pp 75-90

2. Sycara, K., K. Decker, \& D. Zeng (1998) Intelligent Agents in Portfolio Management. In: N. Jennings \& M. Woolridge (eds) Agent Technology: Foundations, Applications, and Markets, Springer, pp. 267-283.

3. Fregene, K., D. Kennedy, and D. Wang (2001) HICA: A Framework for Distributed Multiagent Control, Proc. Int. Conf. on Intelligent Systems and Control, Tampa, USA. pp 187-192

4. Schoonderwoerd, R., O. Holland, \& J. Bruten (1997) Ant-like agents for load balancing in telecommunications networks. In: W. L. Johnson \& B. Hayes-Roth (eds) Proc. 1st Int. Conf. on Autonomous Agents, ACM Press, pp 209-216

5. Chavez, A., A. Moukas, \& P. Maes (1997) Challenger: A multiagent system for distributed resource allocation. In Proc. Int. Conf. on Autonomous Agents, Marina Del Ray,California.

6. Knoblock, C.A., Y. Arens, \& C. Hsu (1994) Cooperating agents for information retrieval. Proc. $2^{\text {nd }}$ Intl. Conf. on Cooperative Information Systems. Univ. of Toronto Press.

7. Lerman, K. (2001) Design and Mathematical Analysis of Agent-based Systems, Lecture Notes in Artificial Intelligence (LNAI 1871), Springer Verlag, pp 222-234

8. Marwala T., P. De Wilde, V. Abramov, N. Szirbik, J. Goossenaerts, L. Correia, P. Mariano, R. Ribeiro (2001) Scalability and optimisation of a committee of agents using genetic algorithm. In Proc. Int. Symp. on Soft Computing and Intelligent Systems for Industry, Paisely, Scotland.

9. Abramov, V.A., N. Szirbik, J.B.M. Goossenaerts, T. Marwala, P. De Wilde, L. Correia, P. Mariano and R. Ribeiro, Ontological Basis for Open Distributed Multi-Agent System, Symposium on Adaptive Agents and Multi-Agent Systems, AISB'01, York, UK, March, 2001.

10. Goossenaerts, J., Pelletier, C. (2002) Ontological Commitment for Participative Simulation. In: H. Arisawa, Y. Kambayashi, V. Kumar, H.C. Mayr, I. Hunt (eds) Conceptual Modeling for New Information Systems Technologies. Lecture Notes in Computer Science 2465, Springer Verlag, pp. 127-140 
11. Hasegawa, A., Kumagai, S., Itoh, K. (2000) Collaboration Task Analysis by Identifying Multi-Context and Collaborative Linkage, Concurrent Engineering Research and Applications Journal, 8(1), pp 100-108

12. Guarino, N. (1997) Some organizing principles for a unified top-level ontology. Internal report 02/97 V3.0, LADSEB-CNR, Italy.

13. Goossenaerts, J.B.M. (2003) Architecting a Ubiquitous \& Model Driven Information Infrastructure (this volume).

14. Heisig, P., Callot, M., Goossenaerts, J., Kosanke, K., Krogstie, J., Stojanovic, N. (2002) Anchoring Knowledge in Business Process Models to support the Interoperability of Virtual Organizations. In: Kosanke, K, Jochem R., Ortiz, A., Nell, J.G. (eds) Enterprise Inter- and Intra Organization Integration: Building International Consensus, Kluwer Academic Publishers.

15. Goossenaerts, J.B.M., Pelletier, C. (2002) Ontology and Enterprise Modelling. In: Eijnatten van, F.M. (ed.) Participative Simulation Environment for Integral Manufacturing Enterprise Renewal. TNO Arbeid, Amsterdam, The Netherlands, pp.4152.

16. Audi, R. (1998) Epistemology - a contemporary introduction to the theory of knowledge, Routledge, London and New York. 